\title{
Infection control and tuberculosis among health care workers in Viet Nam, 2009- 2013: a cross-sectional survey
}

\author{
Edine W. Tiemersma ${ }^{1 *}$, Nguyen Thien Huong ${ }^{2}$, Pham Hoang Yen ${ }^{3}$, Bui Thi Tinh², Tran Thi Bich Thuy ${ }^{3}$, \\ Nguyen Van Hung ${ }^{3}$, Nguyen Thanh Mai', Suzanne Verver', Agnes Gebhard ${ }^{1}$ and Nguyen Viet Nhung ${ }^{3}$
}

\begin{abstract}
Background: Data on tuberculosis (TB) among health care workers (HCW) and TB infection control (TBIC) indicators are rarely available at national level. We assessed multi-year trends in notification data of TB among HCW and explored possible associations with TBIC indicators.

Methods: Notified TB incidence among HCW and 3 other TBIC indicators were collected annually from all 64 provincial and 3 national TB facilities in Vietnam. Time trends in TB notification between 2009 and 2013 were assessed using linear regression analysis. Multivariate regression models were applied to assess associations between the facility-specific 5-year notification rate and TBIC indicators.

Results: Forty-seven (70 \%) of 67 facilities contributed data annually over five years; 15 reported at least one HCW with TB in 2009 compared to six in 2013. The TB notification rate dropped from 593 to 197 per 100,000 HCW ( $p_{\text {trend }}=0.02$ ).

Among 104 TB cases reported, 30 were employed at TB wards, 24 at other clinical wards, ten in the microbiology laboratory, six at the MDR-TB ward, and 34 in other positions.

The proportion of facilities with a TBIC plan and focal person remained relatively stable between $70 \%$ and $84 \%$. The proportion of facilities providing personal protective equipment (PPE) to their staff increased over time. Facilities with a TBIC focal person were 7.6 times more likely to report any TB cases than facilities without a focal person.

Conclusions: The TB notification rates among HCW seemed to decrease over time. Availability of PPE increased over the same period. Appointing a TBIC focal person was associated with reporting of TB cases among HCW. It remains unclear whether TBIC measures helped in reduction of the TB notification rates in HCW.
\end{abstract}

Keywords: Tuberculosis, Health care workers, Viet Nam, Questionnaire, Infection control

\section{Background}

Since long, tuberculosis (TB) is regarded as an occupational risk for health care workers $(\mathrm{HCW})$, and $\mathrm{HCW}$ have been shown to have a higher risk of active TB than the general population in multiple studies [1-4]. However, little is known about the burden of $\mathrm{TB}$ disease among $\mathrm{HCW}$ globally since TB among $\mathrm{HCW}$ is not registered as such in most routine TB surveillance systems. The World Health Organization (WHO) intends to

\footnotetext{
* Correspondence: edine.tiemersma@kncvtbc.org

${ }^{1}$ KNCV Tuberculosis Foundation, Benoordenhoutseweg 46, 2596 BC The

Hague, The Netherlands

Full list of author information is available at the end of the article
}

include an indicator for monitoring of $\mathrm{TB}$ among $\mathrm{HCW}$, which is the ratio of TB notification rate (all forms) in HCW (all staff) over the TB notification rate in the general population, adjusted for age and sex, in future Global TB reports [5].

One of the major objectives of the 2009 "WHO Policy on TB Infection Control in healthcare facilities, congregate settings and households" [6] is to establish effective TB infection control (IC) measures at healthcare facilities. Successful implementation of TBIC measures is important for preventing $\mathrm{HCW}$, patients and visitors from becoming infected with drug sensitive and drug resistant TB [1-4, 7-9]. In addition, monitoring the 
occurrence of active TB among HCW could be a proxy for assessing reduced TB transmission associated with the implementation of TBIC in healthcare facilities [10].

Vietnam is one of the 22 high TB burden countries, with an estimated incidence rate of 144 per 100,000 per year (95\% uncertainty interval, 121-174) in 2013 [5]. To date, no studies have been conducted to assess the burden of active TB disease among $\mathrm{HCW}$ in the country, and the routine, patient based, electronic recording and reporting system does not record $\mathrm{HCW}$ specifically. However, one study comparing TB infection prevalence in one of the four referral hospitals with the adjacent non-TB hospital, showed a high prevalence of latent TB infection among HCW in the TB hospital (38 \%-55 \% in the youngest age group (20-29 years), depending on the method used), and a two times increased TB infection risk compared to $\mathrm{HCW}$ in the same age group in the non-TB hospital. This increased risk may have been due to inadequate IC measures: $\mathrm{HCW}$ and $\mathrm{TB}$ patients used the same (and only) hospital entrance, and only surgical masks, instead of N95 respirators, were available [11].

Vietnam is one of the first high TB incidence countries collecting annual data on $\mathrm{TB}$ among $\mathrm{HCW}$ and other TBIC indicators in all national and provincial TB and lung diseases facilities for at least five years. Here, we report the results of this annual assessment.

\section{Methods}

\section{Aim}

We aimed to assess time trends in the annual notification rates (2009-2013) of TB among HCW in these provincial and national facilities. Also, we explored potential associations between TB notification rates and different TBIC indicators reported.

\section{Design}

To get better insight in the burden of TB among HCW employed in public $\mathrm{TB}$ facilities connected to the National TB Program (NTP) in Vietnam, NTP has developed four main TBIC indicators:

- Proportion of HCW with TB among all HCW per year;

- Proportion of health facilities that have a (valid, i.e., not outdated) TBIC plan;

- Proportion of health facilities that have an appointed TBIC focal person;

- Proportion of HCW working in the multidrug resistant (MDR)-TB ward or in the culture and/or drug susceptibility testing (DST) section of the hospital's laboratory that is provided at least one N95 respirator per week.

Based on these indicators, a short questionnaire was designed in English and translated in Vietnamese. It included additional questions on the characteristics of the TB patients among $\mathrm{HCW}$, the duration of validity of the TBIC plan, the presence of a TBIC focal person and provision of personal protective equipment (PPE) to $\mathrm{HCW}$ working in the culture and/or DST section of the microbiology department and HCW serving in MDR-TB wards. Annually, between 2010 and 2013, the form was sent to all national and provincial TB facilities, requesting the administration department of the hospital to complete the form. Information was collected on the period 2009-2013 by the TBIC focal person, who was usually part of the infection control committee overseeing implementation of IC policies in the hospital. Minor changes were made in the form over the years (see Results section).

\section{Setting}

The provincial facilities for diagnosis and treatment of $\mathrm{TB}$ of all 63 provinces in Vietnam were included in this study: 43 TB and lung diseases hospitals, 18 preventive medicine centers for social diseases control and prevention, two preventive medicine centers, and one TB station. Hanoi both has a provincial TB hospital and a preventive medicine center for social diseases. Also, the three national TB and lung disease hospitals were included so that the total number of health facilities was 67. National hospitals are highly specialized hospitals that provide secondary and tertiary care to patients, most of whom have been referred from district and provincial levels, while provincial TB and lung disease hospitals usually provide secondary care and serve as referral centers for district TB units. The Ministry of Health's circular 18/2009/TT-BYT, published on 14 October 2009, provided guidance in implementation of IC activities in health care facilities and led to the start of IC implementation in all TB services within NTP. Annual medical check-up for HCW is required by law, and involves mandatory X-ray for all those suspected of TB (i.e. with cough of more than two weeks). These check-ups are executed by medical examination facilities, which aggregate all cases diagnosed with any of 34 occupational diseases and report these twice a year to the Ministry of Health. TB is regarded occupational if active disease is found in a person working in a high-risk location (i.e., M. tuberculosis aerosols detected during periodic assessments) for a minimum duration of 6-12 months, depending on the type of TB (12 months for pulmonary, bone/joint, and urinogenital TB, 6 months for other forms of TB).

In Vietnam, Culture/DST rooms in the TB laboratories all use negative pressure. MDR-TB wards in principle use natural ventilation, but if there are less than 12 air changes per hour, natural ventilation is 
combined with an exhaust fan and ultraviolet germicidal irradiation (UVGI).

\section{Definitions}

A health facility was defined as any TB service at provincial or supra-provincial level, as described under Setting.

A HCW was defined as anyone occurring on the payroll of the health facility in the specific year of reporting. This includes staff working in any clinical department, in paramedical sections (such as pharmacy), laboratories, and administrative and other support sections of the health facility.

TB was defined as active TB, and includes pulmonary (new and retreatment) and extra-pulmonary TB. Since reporting was anonymous we could not check whether these patients were notified and/or bacteriologically confirmed, and included any patient reported by the facility.

A TBIC plan is a written protocol for the prompt recognition, separation, provision of services, investigation for TB and referral of patients with suspected or confirmed TB disease in the health facility [12]. The TBIC plan was considered valid if the validity period included the reporting period. Since the TBIC plans were not available to the researchers, no content checks were conducted.

A TBIC focal person is the person in the health facility who is responsible for coordination of all TBIC activities in the health facility.

\section{Ethical issues}

The project was approved by the Research Board of the National Lung Hospital in Hanoi. Only data that had been routinely collected by each health facility were requested. Data on HCW were collected in an aggregated manner. No personal information about HCW was requested. Therefore, no personal informed consent was obtained. All health facilities received a code before analysis. All data were analyzed in such a way that the information presented does not provide sufficient information for the identification of persons.

\section{Data entry and analysis \\ Data entry and validation}

Submitted forms were checked for completeness and consistencies. Inconsistencies and omissions were checked and solved by phone and email with the health facility's contact person. All data were entered in a pre-designed EpiData data entry sheet (www.epidata.dk). The entered data were compared with the paper forms by a person who had not been involved in data entry.

Data analysis was performed in Stata/SE 11.1 for Windows (Stata Corp., College Station, Texas, USA; www.stata.com).

\section{Data analysis}

Notification rates were calculated by dividing the summed number of HCW with TB disease in a specific year by the summed number of HCW working in the health facilities in the same year. It was assumed that each HCW worked in the health facility for a full year. The rates were multiplied by 100,000 to obtain a notification rate per 100,000 HCW per year. Exact binomial $95 \%$ confidence intervals (CIs) were calculated around each annual rate. Time trends were assessed applying random effects negative binomial regression analysis on the annual notification rates between 2009 and 2013 after specifying the data as panel (time series) data.

Health facilities were grouped to assess health facility characteristics as follows: (national) referral centers for TB $(n=4$; including the three national TB hospitals and one provincial TB hospital acting as a tertiary referral center), provincial TB hospitals $(n=43$; including all but one provincial hospitals for $\mathrm{TB}$ and lung diseases), and provincial preventive centers $(n=20$; including 18 preventive medicine center for social diseases control and prevention and two TB stations).

Multivariate regression models were applied to assess the association between the health facility specific 5-year notification rate and health facility characteristics. Because of the (bimodal) distribution characteristics of the 5-year TB notification rates (see Fig. 1), different multivariate regression models were applied. First, a backward stepwise logistic regression model was built in which the dependent variable was either 1 (any TB cases reported between 2009 and 2013) or 0 (no TB cases at all between 2009 and 2013) to account for the $42 \%$ of health facilities reporting no cases between 2009 and 2013. All variables for which the $\mathrm{p}$-value was less than 0.2 were kept in the model. To assess the associations between notification rates and facility characteristics in those facilities from which cases were reported, the TB notification rate was first log-transformed and this transformed notification rate was analyzed using simple linear regression models. Here, backward stepwise linear regression was applied leaving all variables for which the $p$-value was less than 0.2 in the model. Time trends were calculated in- and excluding health facilities that did not provide data for all of the years 2009-2013. A $p$-value smaller than 0.05 was regarded as statistically significant.

\section{Results}

Tuberculosis notification rates among health care workers The number of health facilities responding to the survey varied between 57/67 (85\%) in 2012 and 2013 and 64/ 67 (96\%) in 2011 (Table 1). The number of health facilities reporting at least one $\mathrm{TB}$ case for the respective year of reporting decreased between 2009 and 2013: from $18 / 61(30 \%)$ to $9 / 54(17 \%)(p=0.02)$. 


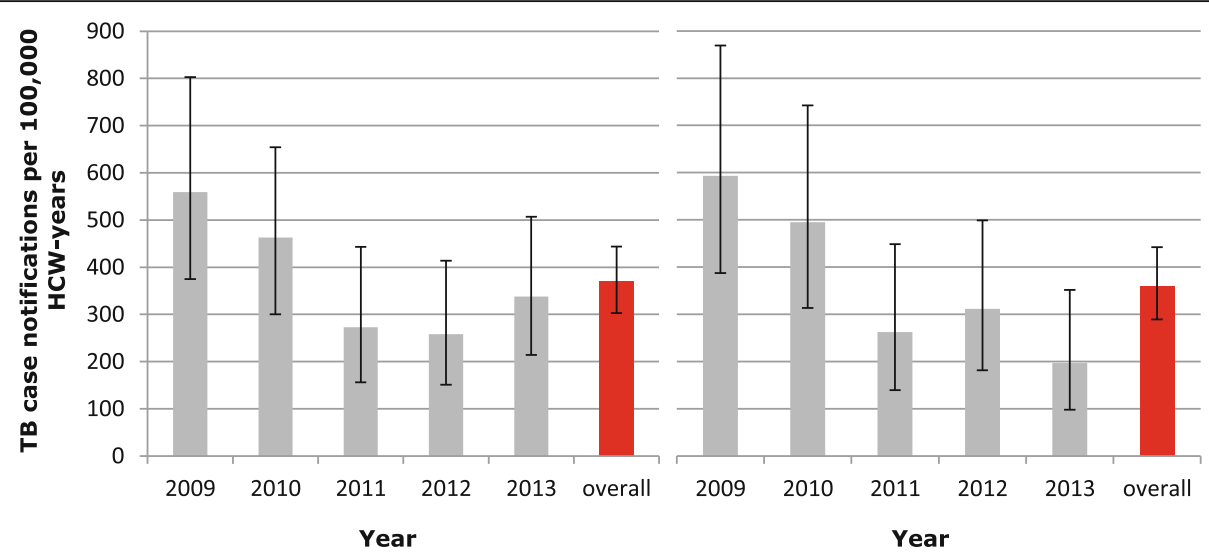

Fig. 1 Reported annual TB case notification rate per 100,000 health care worker (HCW) years. Left panel includes all health facilities providing information on the number of TB cases and the number of HCW in any given year (2009-2013); right panel includes only the health facilities reporting this information for all five years. Error bars depict $95 \%$ confidence intervals

The notification rate of all types of TB dropped from 559 per 100,000 HCW per year in 2009 to 338 per 100,000 HCW per year in 2013 (Fig. 1, left panel).

A time trend in TB notification rates was calculated using data from 47 facilities that reported data for all five years. In these facilities, the notification rates dropped significantly from 593 (95\%CI, 388-869) per $100,000 \mathrm{HCW}$ years in 2009 to 197 (95\% CI, 98-352) per $100,000 \mathrm{HCW}$ years in $2013,(p=0.02$; betacoefficient $-0,2,95 \% \mathrm{CI},-0.36$ to -0.03 ; Fig. 1 , right panel). We repeated this analysis excluding four health facilities with a reported increase of $>100 \%$ in health staff within one year. This did not change the results: the decrease remained statistically significant $(p=0.04$; data not shown).

Overall, $56 \%$ of the reported TB cases were registered new pulmonary TB cases, $6 \%$ were previously treated pulmonary $\mathrm{TB}$ cases, and $38 \%$ were registered with extra-pulmonary TB. The distribution remained more or less stable over the years.

Of a total of 104 (95\%) TB cases for whom the working position was recorded, 30 (29\%) were reported among staff employed at TB wards, 24 (23\%) among staff from other (non-TB) clinical wards, 10 (10\%) among microbiology laboratory staff, six (6\%) among staff working at the MDR-TB ward, and 34 (33\%) among staff working in other positions. It remains unknown how the number of TB cases reported relates to the number of staff members per working position, as staff counts per working position were not included in the data collection form for feasibility reasons.

Over the years, especially among workers in the microbiology departments a reduction of TB notification rates was seen (beta-coefficient -0.61 (95 \% CI -1.18 to -0.03$), p=0.04$ ).

\section{TBIC measures and TB among health care workers}

In the period of 2009-2011, 48 of 64 health facilities (75 \%) reported having a TBIC plan available; this plan was reported to be available by 45 of 56 facilities $(80 \%)$ reporting over the period in 2012-2013 (Table 2).

In a sensitivity analysis, taking only those health facilities into account reporting on this indicator both in the period 2009-2011 and 2012-2013 $(n=53)$, the proportion of facilities with a TBIC plan also remained stable (41 (77 \%) had a plan in 2009-2011 and 43 (81\%) had one in 2012-2013). The median period of validity of a TBIC plan was three years (interquartile range, 2-5 years), with a maximum of 13 years.

It is not known which TBIC plans had been based on (inter)national guidelines, and how many of these had been made with input from TBIC experts.

The proportion of facilities reporting to have an appointed TBIC focal person was $83 \%(52 / 63$ facilities) for 2009-2011 and 84\% (47/56 facilities) for 2012-2013, and $73 \%$ taking only the 52 health facilities into account that reported on this indicator for both periods (Table 2).

N95 respirators were provided in the majority of microbiology laboratories providing culture (with or without DST) and health facilities with an MDR-TB ward (Table 2). The proportion of such facilities having PPE available for their staff increased between 20092011 and 2012-2013 (from $61 \%$ to $90 \%, p=0.02$ for culture/DST laboratories, and from $67 \%$ to $100 \%, p=$ 0.01 for MDR-TB wards), assuming those reporting are representative for all facilities with an MDR-TB ward and/or a culture/DST laboratory.

Only six health facilities had not implemented any TBIC measures over the reporting period; these include one TB unit in a provincial hospital and five preventive medicine centers. Together, these facilities 
Table 1 Tuberculosis (TB) cases among health care workers in provincial and national TB facilities, 2009-2013

\begin{tabular}{|c|c|c|c|c|c|c|}
\hline \multirow[t]{2}{*}{ Characteristic } & \multicolumn{5}{|c|}{ Year of report } & \multirow[t]{2}{*}{ Total } \\
\hline & 2009 & 2010 & 2011 & 2012 & 2013 & \\
\hline Number of health facilities sending report & 62 & 63 & 64 & 57 & 57 & \\
\hline Number of HCW working in these facilities & 5,225 & 5,455 & 5,940 & 6,580 & 6,811 & \\
\hline Number of health facilities reporting the number of TB cases among HCW & 61 & 61 & 62 & 54 & 54 & \\
\hline Number of HCW in facilities reporting the number of TB cases & 5,183 & 5,396 & 5,870 & 6,580 & 6,811 & 29,840 \\
\hline Number of facilities reporting at least one TB case & 18 & 18 & 12 & 13 & 9 & $39^{b}$ \\
\hline Number of TB cases (all forms) & 29 & 25 & 16 & $17^{c}$ & 23 & 110 \\
\hline Notification rate of all TB/100,000 HCW-years & 560 & 463 & 273 & 258 & 338 & 369 \\
\hline $95 \%$ confidence interval & $375-803$ & $300-684$ & $156-443$ & $151-414$ & $214-507$ & $303-444$ \\
\hline \multicolumn{7}{|l|}{ Among health facilities reporting data for 5 consecutive years $(n=47)$} \\
\hline Number of HCW working in these facilities & 4,382 & 4,648 & 4,954 & 5,453 & 5,594 & 25,031 \\
\hline Number of facilities reporting at least one TB case & 15 & 16 & 10 & 12 & 6 & $30^{\mathrm{b}}$ \\
\hline Number of TB cases (all forms) & 26 & 23 & 13 & 17 & 11 & 90 \\
\hline Notification rate of all TB/100,000 HCW-years & 593 & 495 & 262 & 312 & 197 & 360 \\
\hline $95 \%$ confidence interval & $388-868$ & $314-742$ & $140-448$ & $182-499$ & $98-352$ & $289-442$ \\
\hline Number of facilities with complete data for type of TB & 61 & 60 & 62 & 52 & 52 & \\
\hline Number of TB cases in these facilities & 29 & 24 & 16 & 15 & 17 & $101(92 \%)$ \\
\hline \multicolumn{7}{|l|}{ Number of TB cases by type of TB: } \\
\hline New pulmonary TB & 19 & 10 & 9 & 8 & 11 & $57(56 \%)$ \\
\hline Previously treated pulmonary TB & 3 & 1 & 1 & 1 & 0 & $6(6 \%)$ \\
\hline Extra-pulmonary TB & 7 & 13 & 6 & 6 & 6 & $38(38 \%)$ \\
\hline Number facilities with complete data for job category of HCW with TB & 61 & 61 & 62 & 53 & 54 & \\
\hline Number of TB cases in these facilities & 27 & 24 & 15 & 16 & 22 & $104(95 \%)$ \\
\hline \multicolumn{7}{|l|}{ Number of TB cases among HCW employed in/at: } \\
\hline microbiology department $^{d}$ & 6 & 1 & 2 & 0 & 1 & $10(10 \%)$ \\
\hline TB wards & 8 & 8 & 2 & 6 & 6 & $30(29 \%)$ \\
\hline MDR-TB ward ${ }^{d}$ & 1 & 2 & 2 & 1 & 0 & $6(6 \%)$ \\
\hline other (non-TB) clinical wards & 3 & 4 & 2 & 3 & 12 & $24(23 \%)$ \\
\hline other positions & 9 & 9 & 7 & 6 & 3 & $34(33 \%)$ \\
\hline
\end{tabular}

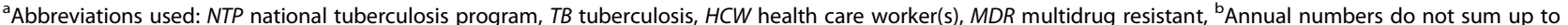
the total as some health facilities reported at least one TB case in more than one of the five years; ' ${ }^{\circ}$ One provincial TB hospital was excluded since one TB case was reported for 2012 without providing the number of health care workers in that year, so that notification rate cannot be calculated; ${ }^{{ }}$in total, 26 facilities had a culture laboratory and 25 hospitals reported having an MDR-TB ward

reported only one extra-pulmonary TB case in five years' time. Having only limited data on TBIC measures available, we did not find an effect of any improvements in TBIC measures (defined as newly implementing a TBIC plan or appointing a TBIC person or starting the use of PPE in MDR-TB wards and culture laboratory sections) on the TB notification rates (data not shown). Among the facilities with MDR-TB wards and/or culture sections, the 5-year notification rates seemed lower in those with reported provision of N95 respirators (354/100,000 HCWyears, $95 \%$ CI 277-445) than in those without (448/ 100,000 HCW-years, $95 \%$ CI 180-921), but this difference was not statistically significant.
The notification rates were lower in those facilities that had no TBIC focal person compared to facilities with a TBIC focal person (Kruskal-Wallis ranksum test $p$-value, $p=0.08$ for 2009-2011 and $p=0.04$ for 2012-2013).

\section{Health facility characteristics and TB among health care workers}

Table 3 presents notification rates by facility type and geographical zone (all reports of the health facilities received from 2009-2013 included). The notification rate was near-to-significantly higher in the referral centers compared to provincial health facilities $(p=0.06)$, with a conditional maximum likelihood estimate (CMLE) of the 
Table 2 TBIC indicators reported by provincial and national TB facilities to the NTP, 2009-2013 ${ }^{\text {a }}$

\begin{tabular}{|c|c|c|c|c|}
\hline \multirow[t]{2}{*}{ Indicator } & \multirow[t]{2}{*}{ Number of facilities } & \multicolumn{3}{|c|}{ Period of reporting } \\
\hline & & $2009-2011$ & $2012-2013$ & Total (with information/answer 'yes' for both periods) \\
\hline \multicolumn{5}{|l|}{ TBIC plan } \\
\hline & Number (\%) with information & 64 & 56 & 53 \\
\hline & Number (\%) yes & $48(75 \%)$ & $45(80 \%)$ & $37(70 \%)$ \\
\hline \multicolumn{5}{|c|}{ TBIC focal person } \\
\hline & Number (\%) with information & 63 & 56 & 52 \\
\hline & Number (\%) yes & $52(83 \%)$ & $47(84 \%)$ & $38(73 \%)$ \\
\hline \multicolumn{5}{|c|}{ PPE provided to staff } \\
\hline & Number with TB culture lab & 23 & 24 & 20 \\
\hline & Number of those answering the question & 23 & 21 & 19 \\
\hline & Number (\%) of those with PPE & $14(61 \%)$ & $19(90 \%)$ & $11(55 \%)$ \\
\hline & Number with MDR-TB ward & 15 & 24 & 14 \\
\hline & Number of those answering the question & 15 & 22 & 12 \\
\hline & Number (\%) of those with PPE & $10(67 \%)$ & $22(100 \%)$ & $9(64 \%)$ \\
\hline
\end{tabular}

${ }^{a}$ Abbreviations used: TB tuberculosis, $I C$ infection control, NTP national tuberculosis program, MDR multidrug resistant, $P P E$ personal protective equipment

rate ratio of 1.5 (95\% CI 0.99-2.2). The TB notification rate in Southern health facilities was higher than in Northern, and statistically significantly higher than in Central facilities $(p=0.002$; CMLE rate ratio $4.9,95 \% \mathrm{CI}$ 1.7-20). The notification rate in the North was also higher than in the Central zone $(p=0.02$; CMLE rate ratio 3.7, $95 \%$ CI 1.3-14.7) (Table 3).

Among the provincial TB hospitals, there was a significant inverse linear association between the number of HCW employed and 5-year TB notification rate ( $p=$ $0.001 ; \beta$-coefficient $=0.8$, standard error $=0.2$; Fig. 2 ) .

Facilities with an appointed IC focal person were 7.6 times more likely to report any TB cases than facilities that had no focal person (Table 4). Compared to health facilities in Central Vietnam, facilities in North Vietnam were 10 times more likely to report a TB case (Table 4).

\section{Discussion}

Main results of the study

Over the reporting period (2009-2013), the rate of all types of TB reported dropped from 559 per to 338 per $100,000 \mathrm{HCW}$-years. Including only the 47 health facilities reporting for five consecutive years, the notification rates dropped from 593 to 197 per 100,000 HCW years (test for trend, $p=0.02$ ). While this is a marked decline, in 2013 the notification rate among HCW was still almost twice as high as in the general population (which was $111 / 100,000$ in 2013 [5]). This may in part reflect better access to screening and awareness about the occupational risk of $\mathrm{TB}$ transmission, but will also reflect the higher risk of getting TB among HCW, which is consistently reported in almost all published literature on this topic $[1,2,4,13]$. The most recent systematic review reported a relative risk (RR) of active TB disease for HCW

Table 3 Notification rate of all types of tuberculosis per 100,000 health care worker-years, $2009-2013^{a}$

\begin{tabular}{|c|c|c|c|c|c|}
\hline \multirow[t]{2}{*}{ Type of facility } & \multirow{2}{*}{$\begin{array}{l}\text { Number of } \\
\text { facilities }^{b}\end{array}$} & \multirow{2}{*}{$\begin{array}{l}\text { Number of } \\
\text { HCW-years }\end{array}$} & \multirow{2}{*}{$\begin{array}{l}\text { Number } \\
\text { of TB } \\
\text { cases }^{b}\end{array}$} & \multicolumn{2}{|c|}{ Tuberculosis notifications, all types, per 100,000 HCW-years } \\
\hline & & & & Rate & $(95 \% \mathrm{Cl})^{\mathrm{c}}$ \\
\hline \multicolumn{6}{|l|}{ Type of facility } \\
\hline Referral hospitals for TB \& lung diseases & 4 & 8,257 & 40 & 484 & $(346-660)$ \\
\hline Provincial hospitals \& centers for TB \& lung diseases & 42 & 18,388 & 60 & 326 & $(249-420)$ \\
\hline Provincial preventive centers ${ }^{d}$ & 20 & 3,153 & 10 & 317 & $(152-583)$ \\
\hline \multicolumn{6}{|l|}{ Region } \\
\hline North & 32 & 17,626 & 63 & 357 & $(275-457)$ \\
\hline Central & 12 & 3,068 & 3 & 98 & $(20-286)$ \\
\hline South & 22 & 9,157 & 44 & 481 & $(349-645)$ \\
\hline
\end{tabular}

${ }^{a}$ Abbreviations used in this Table: TB tuberculosis, $H C W$ health care worker, $C l$ confidence interval. ${ }^{\mathrm{b}}$ One provincial TB hospital in Central Vietnam was excluded since one TB case was reported for 2012 without providing the number of health care workers in that year, so that notification rate cannot be calculated. ${ }^{\mathrm{C}} 95 \%$ binomial exact confidence intervals are displayed. ${ }^{\mathrm{d}}$ These include centers for social disease prevention $(n=18)$ and medical preventive centers $(n=2)$ 


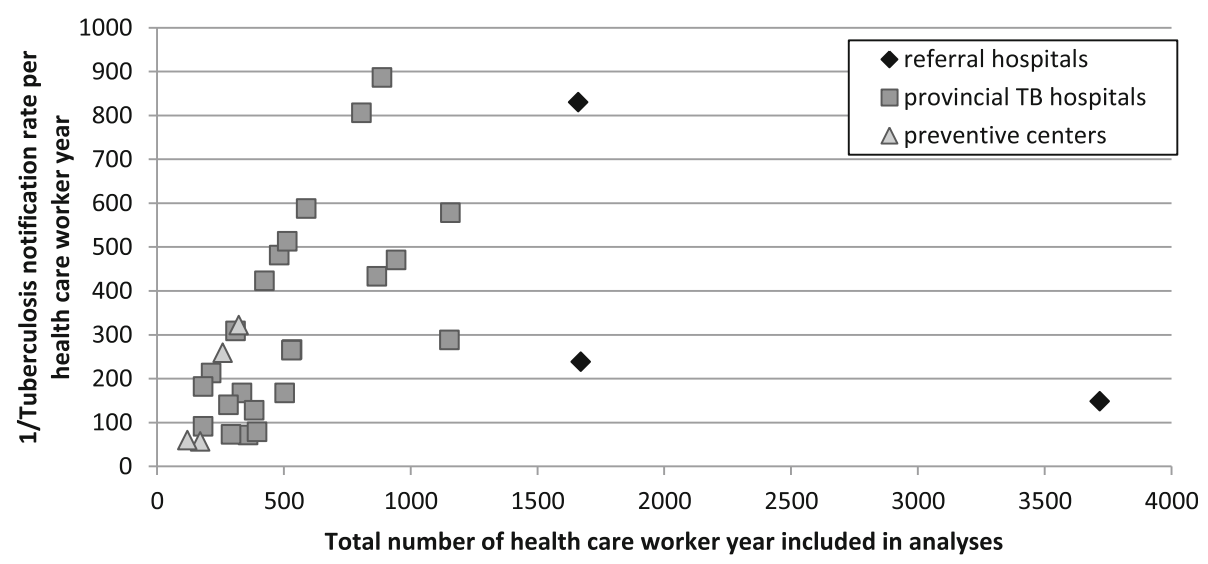

Fig. 2 Five-year health care worker (HCW) TB notification rate versus number of HCW employed. Each marker depicts one health facility; the different types of markers refer to the different types of health facilities included

compared to the general population of 3.7 (95\% CI 2.94.5) with RRs ranging from 1.2 to 14.7 depending on the setting [1]. It should be noted however that these RRs were not corrected for age and sex, since this information is often not available. Between 2009 and 2013, there was a statistically significant decline $(p=0.04)$ in the TB notification rates among staff working in TB laboratories. This decline may be the result of increased protection and awareness of staff, the availability of biosafety cabinets and improved laboratory practices as a result of continued training activities. Though a decline was also seen among staff working in MDR-TB departments, this was much weaker and failed to reach statistical significance. Notably, the proportion of health facilities providing PPE to staff increased from $61 \%$ in 2011 to $79 \%$ in 2013 for staff in MDR-TB wards ( $p=$ $0.17)$ and from $67 \%$ to $92 \%(p=0.048)$ for staff in the culture/DST section of the microbiology laboratories. It should be noted that the number of TB patients found in non-TB related departments remained relatively high, and this is in line with results found by an earlier review [2]. Also it should be noted that the available data (all age/sex classes) shows no decline over time in notification rates among the general population during the study period.

Only a few studies have measured the effect of TBIC measures on the TB burden among HCW $[7,8,10,14-16]$ and from three of these studies, a decrease in latent $\mathrm{TB}$

Table 4 Association between health facility characteristics (2013) and $\geq 1$ tuberculosis case reported between 2009 and 2013

\begin{tabular}{|c|c|c|c|c|}
\hline \multirow[t]{2}{*}{ Characteristic } & \multicolumn{2}{|l|}{ Univariate } & \multicolumn{2}{|l|}{ Multivariate } \\
\hline & Odds Ratio & $95 \%$ Confidence Interval & Odds Ratio & $95 \%$ Confidence Interval \\
\hline \multicolumn{5}{|l|}{ Type of health facility } \\
\hline Policlinic only & 1 & REF & & \\
\hline Additional inpatient treatment & 6.9 & $2.1-23.1$ & & \\
\hline HCW-years (increase with 50 HCW-years ${ }^{a}$ ) & 1.3 & $1.1-1.5$ & 1.1 & $0.96-1.4$ \\
\hline \multicolumn{5}{|l|}{ Location of the facility in: } \\
\hline Central Vietnam & 1 & REF & 1 & REF \\
\hline North Vietnam & 12.0 & $2.5-58.5$ & 10.0 & $1.6-61.2$ \\
\hline South Vietnam & 2.5 & $0.5-11.8$ & 4.4 & $0.8-24.6$ \\
\hline Presence of culture laboratory & 2.7 & $0.9-7.9$ & & \\
\hline Presence of MDR-TB ward & 2.7 & $0.9-7.9^{b}$ & & \\
\hline (TB)-IC plan present & 3.8 & $1.1-12.8$ & & \\
\hline Appointed focal person for (TB)-IC available & 8.6 & $1.7-44.8$ & 7.6 & $1.2-47.5$ \\
\hline PPE available in culture labs/MDR-TB ward & 3.7 & $1.2-11.1$ & & \\
\hline
\end{tabular}


infection was reported, but not a decrease in TB disease. One cross-sectional study only reported about TB disease [10]. A weak association was found between reported IC measures (especially environmental measures) and TB disease incidence, but it disappeared in the multivariable analysis. About $75 \%$ of health facilities included in our report had a TBIC plan and an appointed TBIC focal person. These proportions remained stable over time. Presence of a TBIC focal person in the facility generally was associated with a higher probability of reporting any TB cases (adjusted Odds Ratio $=7.695 \%$ CI 1.2-47.7). Similarly, presence of a TBIC plan was associated with finding one or more TB-cases among staff members. This may be the effect of less active case finding and reporting from facilities without a TBIC focal person as compared to facilities with a TBIC focal person. The alternative explanation may be that those facilities with more TB cases among HCW may be encouraged to appoint a TBIC focal person (reverse causality). The information that we collected was probably not specific enough to detect any effect of TBIC measures. Further, confidence intervals are wide since we conducted ecological analysis, with one data point for each health facility. Presence of a TBIC plan alone likely does not contribute to reduced transmission of TB or increased case finding within a facility. This information was not available from the routine reports unfortunately and additional data should be collected about TBIC practices.

Although there is evidence that implementation of IC measures reduces the burden of TB [2, 4], few studies evaluated the impact of TBIC measures in low and middle income countries (LMIC). One study found no effect on TB disease incidence [14] and two showed a reduction in tuberculin skin test conversion rates in $\mathrm{HCW}$ $[7,8,15]$. In LMIC, even low-cost strategies are rarely implemented $[3,15,17]$ though recently IC has gained more attention and more is being done to protect $\mathrm{HCW}$ from TB infection and disease.

Not surprisingly, larger facilities (in terms of number of $\mathrm{HCW}$ ) were more likely to report any TB cases. However, for those facilities in which TB cases were notified, the notification rates decreased with increasing facility size (rate ratio for an increase in size with $50 \mathrm{HCW}$ years: $0.94, p=0.01$ ). This decline in notification rates may be because many of the larger hospitals are tertiary care facilities which tend to pay more attention to TBIC measures, as national implementation of such measures usually starts in tertiary care hospitals which also have more resources available than other hospitals.

Provincial TB hospitals reported lower TB notification rates $(329 / 100,000 \mathrm{HCW}$-years) when compared to other types of facilities (notably national/regional hospitals with a notification rate of 484/100,000 HCW-years). This may be explained by presence of more difficult-totreat TB patients and conduct of higher-risk procedures in the larger hospitals, such as bronchoalveolar lavage and pneumectomy, and culture and DST in laboratories, but also by better awareness among HCW about the risks of TB transmission leading to better adherence to annual screening procedures, and more resources facilitating reporting of TB cases. However, more data are needed to learn which of these explanations contributes most.

The probability of reporting any $\mathrm{TB}$ cases was lower in Central than in North Vietnam (where two of the three regional and the national TB hospital are located). This association may reflect the underlying TB burden in the general population. The TB prevalence survey found a lower prevalence in Central Vietnam as compared to North and South Vietnam $(p<0.05)$ [18], and the data also match the overall TB notification data of NTP [19].

\section{Limitations}

The data presented in this report was collected routinely from all provincial and supra-provincial facilities reporting to the NTP in Vietnam. To our knowledge, this information is rather unique and Vietnam is one of the few countries in the region collecting any indicator data on TBIC practices and on TB cases among $\mathrm{HCW}$. However, routine collection of data on just a few indicators understandably has its limitations as much less information can be collected than is usually done in research settings.

First of all, this concerns indicators aggregated by health facility and data can thus not be linked to individual characteristics such as sex, age, working experience, and job type as has been done by others [10]. Also, comparison with the notification rates in the general population was impossible since the age and sex composition of our study population was unknown. Local laws prescribe aggregate data collection of TB among $\mathrm{HCW}$. Moreover, TB is highly stigmatized in Vietnam, also among $\mathrm{HCW}$, and therefore it was considered inappropriate to collect details that could lead to identification of HCW.

The data only include TB cases known to the health facility administration. In Vietnam, TB is still stigmatized [20] and this may cause HCW to seek diagnosis and treatment elsewhere. On the other hand, annual health screening is mandatory and includes chest X-ray imaging. It is known that $\mathrm{X}$-ray screening is a sensitive method to detect pulmonary TB, also in the absence of TB symptoms [21]. This mandatory system also implies that these notification data were derived from active case finding, which is not comparable to the passive case finding strategies used in Vietnam to diagnose TB.

TB infection (measured by tuberculin skin test or Quantiferon test-conversion) better reflects recent transmission of Mycobacterium tuberculosis than TB disease 
notification since progression to $\mathrm{TB}$ disease may take several years and depends on other factors such as immune status of the host [22]. It may take time until changes in transmission will be measurable as changes in notification. Besides that, in countries with a generalized TB epidemic, transmission is likely to occur also in community settings [23-25].

We analyzed reported information that was not checked by the researchers on site. For example, we did inquire whether a TBIC plan and a focal person were available in the health center and whether N95 respirators were being distributed to the staff. However, though this provided some information, this does not necessarily mean that plans are implemented as they should and respirators are (always) used appropriately when guidelines prescribe so, especially if appropriate training on the importance of $\mathrm{TBIC}$ and its practical implementation is lacking.

This effort is a repetitive, low-workload activity, recording information on a minimum number of TBIC indicators, meeting the WHO recommendation to specifically include notifications of TB among $\mathrm{HCW}$. The minimum dataset did not include the number of staff and TB patients in each job category, completeness of HCW screening and notification, occurrence of MDR-TB, and whether it concerned nosocomial or community-based transmission. To enable assessment of the implementation of TBIC measures, routine on-site monitoring is needed. To help countries set up a standard routine $\mathrm{TB}$ monitoring system among $\mathrm{HCW}$ a guide was developed by an international group of experts under TB CARE I [26]. To assess the effect of TB IC measures on nosocomial TB transmission, sophisticated studies would be needed, including careful assessment of potential epidemiological links and genotyping of TB strains isolated from patients using advanced molecular typing methods, preferably whole-genome sequencing [27].

Finally, presently there are no standardized ways for measuring occupational risk of TB infection and disease in high TB incidence populations (such as the Vietnamese population), or for assessing the implementation of TBIC measures [10]. Repeated surveys could be used to measure and compare infection rates in healthcare workers [28].

\section{Conclusion}

Though the notification rate among HCW had dropped significantly from $593 / 100,000$ in 2009 to $197 / 100,000^{*} y$ in 2013, TB notification rates in public TB facilities were almost twice as high as in the general population (111/ $100,000 * y ; p=0.02)$. There was a decline in the proportion of TB cases among $\mathrm{HCW}$ occurring among those employed in TB sections of the health facility. Around $80 \%$ of the 67 health facilities included had a TBIC plan and a TBIC focal person. Facilities with a TBIC focal person and/or a TBIC plan were more likely to report any TB cases than facilities without such a person.

Vietnam is one of the few countries in the developing world that has set up this routine reporting of $\mathrm{TB}$ among $\mathrm{HCW}$ and thereby shows that occupational risks of TB transmission to $\mathrm{HCW}$ are taken seriously. Although data on TBIC indicators is now routinely collected, more detailed information is needed to assess risk factors for TB disease among $\mathrm{HCW}$ and the impact of TBIC measures. Since TB incidence among HCW is higher than that in the general position, implementation of TBIC measures needs continued attention.

\section{Abbreviations \\ Cl: Confidence interval; CMLE: Conditional maximum likelihood estimate; DST: Drug susceptibility testing; HCW: Health care worker(s); IC: Infection control; LMIC: Low- and middle income countries; MDR: Multidrug resistant; NTP: National tuberculosis program; PPE: Personal protective equipment; RR: Relative risk; TB: Tuberculosis; TBIC: Tuberculosis infection control; UVGI: Ultraviolet germicidal irradiation; WHO: World Health Organization}

\section{Acknowledgments}

We thank the administrators of all health facilities who kindly provided the data needed for this project. We are grateful to the staff who entered all data in EpiData. Dr Nguyen Binh Hoa from NTP Vietnam is thanked for providing notification data. The authors also thank Dr Max Meis from KNCV Tuberculosis Foundation for collaboration and constructive comments during all phases of the project. This study was made possible by the generous support of the American people through the United States Agency for International Development (USAID). The contents are the responsibility ethic of TB CARE I and do not necessarily reflect the views of USAID or the United States Government.

\section{Funding}

The Global Health Bureau, Office of Health, Infectious Disease and Nutrition (HIDN), United States Agency for International Development (USAID), financially supports this publication through TB CARE I under the terms of Agreement No. AID-OAA-A-10-00020. This publication is made possible by the generous support of the USAID. The contents are the responsibility of TB CARE I and do not necessarily reflect the views of USAID or the United States Government.

\section{Availability of data and materials}

The database on which the analyses of this paper were based is the property of NTP VietNam. The director of the NTP can be contacted for more information about the availability of the database.

\section{Authors' contributions}

EWT, NTH, PHY, AG and NVN conceived and designed the work described in this paper. NTH, PHY, TTBT, and NVH designed the data collection tool. NTH, $\mathrm{PHY}$ and BTT collected the data. EWT and SV conducted the data analysis and interpreted the data. EWT, NTH, SV and AG were involved in drafting the manuscript. All authors approved the final submitted version of the manuscript.

\section{Competing interests}

The authors declare that they have no competing interests.

\section{Consent for publication}

Not applicable.

\section{Ethics approval and consent to participate}

The project was approved by the Research Board of the National Lung Hospital in Hanoi. Only data that had been routinely collected by each health facility were requested. Data on HCW were collected in an aggregated manner. No personal information about HCW was requested. Therefore, no personal informed consent was obtained. All health facilities 
received a code before analysis. All data were analyzed in such a way that the information presented does not provide sufficient information for the identification of persons

\section{Author details}

${ }^{1}$ KNCV Tuberculosis Foundation, Benoordenhoutseweg 46, 2596 BC The Hague, The Netherlands. ${ }^{2}$ KNCV Tuberculosis Foundation Vietnam Country Office, 130 Mai Anh Tuan Street, Hanoi, Vietnam. ${ }^{3}$ National Tuberculosis Control Program of Vietnam, 463 Hoang Hoa Tham, Hanoi, Vietnam. ${ }^{4}$ Pham Ngoc Thach Hospital, 120 Hong Bang, Ho Chi Minh City, Vietnam.

\section{Received: 27 April 2016 Accepted: 29 October 2016}

Published online: 10 November 2016

\section{References}

1. Baussano I, Nunn P, Williams B, Pivetta E, Bugiani M, Scano F. Tuberculosis among health care workers. Emerg Infect Dis. 2011;17:488-94.

2. Joshi R, Reingold AL, Menzies D, Pai M. Tuberculosis among health-care workers in low- and middle-income countries: a systematic review. PLoS Med. 2006;3:e494.

3. World Health Organization, International Labour Organization. Joint WHO/ ILO policy guidelines on improving health worker access to prevention, treatment and care services for HIV and TB. Geneva: World Health Organization; 2010.

4. Menzies D, Joshi R, Pai M. Risk of tuberculosis infection and disease associated with work in health care settings. Int J Tuberc Lung Dis. 2007:11: 593-605.

5. World Health Organization. Global tuberculosis report 2014. Geneva: World Health Organization; 2014.

6. World Health Organization. WHO policy on TB infection control in healthcare facilities, congregate settings and households. Geneva: World Health Organization; 2009.

7. Yanai H, Limpakarnjanarat K, Uthaivoravit W, Mastro TD, Mori T, Tappero JW Risk of Mycobacterium tuberculosis infection and disease among health care workers, Chiang Rai, Thailand. Int J Tuberc Lung Dis. 2003;7:36-45.

8. Roth VR, Garrett DO, Laserson KF, Starling CE, Kritski AL, Medeiros EA, et al. A multicenter evaluation of tuberculin skin test positivity and conversion among health care workers in Brazilian hospitals. Int J Tuberc Lung Dis. 2005;9:1335-42.

9. da Costa PA, Trajman A, Mello FC, Goudinho S, Silva MA, Garret D, et al. Administrative measures for preventing Mycobacterium tuberculosis infection among healthcare workers in a teaching hospital in Rio de Janeiro, Brazil. J Hosp Infect. 2009:7:57-64.

10. Claassens MM, van Schalkwyk C, du Toit E, Roest E, Lombard CJ, Enarson DA, et al. Tuberculosis in healthcare workers and infection control measures at primary healthcare facilities in South Africa. PloS One. 2013;8:e76272.

11. Lien LT, Hang NT, Kobayashi N, Yanai H, Toyota E, Sakurada S, et al. Prevalence and risk factors for tuberculosis infection among hospital workers in Hanoi, Viet Nam. PLoS One. 2009;4:e6798.

12. World Health Organization. Guidelines for the prevention of tuberculosis in health-care facilities in resource- limited settings. Geneva: World Health Organization; 1999

13. Menzies D, Fanning A, Yuan L, Fitzgerald M. Tuberculosis among health care workers. N Engl J Med. 1995;332:92-8.

14. Harries AD, Hargreaves NJ, Gausi F, Kwanjana JH, Salaniponi FM. Preventing tuberculosis among health workers in Malawi. Bull World Health Organ. 2002;80:526-31.

15. Wenger PN, Otten J, Breeden A, Orfas D, Beck-Sague CM, Jarvis WR. Control of nosocomial transmission of multidrug-resistant Mycobacterium tuberculosis among healthcare workers and HIV-infected patients. Lancet. 1995;345:235-40

16. Blumberg HM, Watkins DL, Berschling JD, Antle A, Moore P, White N, et al. Preventing the nosocomial transmission of tuberculosis. Ann Intern Med 1995;122:658-63.

17. Pai M, Kalantri S, Aggarwal AN, Menzies D, Blumberg HM. Nosocomial tuberculosis in India. Emerg Infect Dis. 2006;12:1311-18.

18. Hoa NB, Sy DN, Nhung NV, Tiemersma EW, Borgdorff MW, Cobelens FG. National survey of tuberculosis prevalence in Viet Nam. Bull World Health Organ. 2010;88:273-80
19. Vree M, Hoa NB, Sy DN, Co NV, Cobelens FG, Borgdorff MW. Low tuberculosis notification in mountainous Vietnam is not due to low case detection: a cross-sectional survey. BMC Infect Dis. 2007;7:109.

20. Fox GJ, Loan LP, Nhung NV, Loi NT, Sy DN, Britton WJ, Marks GB. Barriers to adherence with tuberculosis contact investigation in six provinces of Vietnam: a nested case-control study. BMC Infect Dis. 2015;15:103.

21. Hoa NB, Cobelens FG, Sy DN, Nhung NV, Borgdorff MW, Tiemersma EW. Yield of interview screening and chest X-ray abnormalities in a tuberculosis prevalence survey. Int J Tuberc Lung Dis. 2012;16:762-7.

22. Salgame P, Geadas C, Collins L, Jones-Lopez E, Ellner JJ. Latent tuberculosis infection - Revisiting and revising concepts. Tuberculosis (Edinb). 2015;95: 373-84

23. Buu TN, van Soolingen D, Huyen MN, Lan NN, Quy HT, Tiemersma EW, et al. Tuberculosis acquired outside of households, rural Vietnam. Emerg Infect Dis. 2010;16:1466-8.

24. Verver S, Warren RM, Munch Z, Richardson M, van der Spuy GD, Borgdorff MW, et al. Proportion of tuberculosis transmission that takes place in households in a high-incidence area. Lancet. 2004;363:212-4.

25. Brooks-Pollock E, Becerra MC, Goldstein E, Cohen T, Murray MB. Epidemiologic inference from the distribution of tuberculosis cases in households in Lima, Peru. J Infect Dis. 2011;203:1582-9.

26. TB CARE I. Guide on the monitoring of TB disease incidence among health care workers. The Hague: TB CARE l; 2013. http://www.challengetb.org/ publications/tools/hss/HCW_TB_Incidence_Measuring_Guide.pdf . Accessed 15 Aug 2016

27. Luo T, Yang C, Peng Y, Lu L, Sun G, Wu J, et al. Whole-genome sequencing to detect recent transmission of Mycobacterium tuberculosis in settings with a high burden of tuberculosis. Tuberculosis (Edinb). 2014;94:434-40.

28. TB CARE I. Guide to measure the prevalence of active TB disease among health care workers. The Hague: TB CARE I; 2012. http://www.tbcare1.org/ publications/toolbox/tools/hss/HCW_TB_Prevalence_Measuring_Guidelines. pdf . Accessed 18 Nov 2014

\section{Submit your next manuscript to BioMed Central and we will help you at every step:}

- We accept pre-submission inquiries

- Our selector tool helps you to find the most relevant journal

- We provide round the clock customer support

- Convenient online submission

- Thorough peer review

- Inclusion in PubMed and all major indexing services

- Maximum visibility for your research

Submit your manuscript at www.biomedcentral.com/submit 